\title{
Neural networks approach v/s Algorithmic approach : A study through pattern recognition
}

\author{
Namrata Aneja* \\ Dyal Singh College, Karnal -132001 India
}

\begin{abstract}
There is a great scope of expansion in the field of Neural Network, as it can be viewed as massively parallel computing systems consisting of an extremely large number of simple processors with many interconnections. NN models attempt to use some organizational principles in a weighted directed graphs in which nodes are artificial neurons and directed edges are connections between neuron outputs and neuron inputs. The main characteristic of neural network is that they have the ability to learn complex non- linear input output relationships. A single artificial neuron is a simulation of a neuron (basic human brain cell) and scientists have tried to emulate the neuron in a form of artificial neuron called perceptron. Pattern recognition is one of the areas where the neural approach has been successfully tried. This study is concerned to see the journey of pattern recognition from algorithmic approach to neural network approach.
\end{abstract}

\section{INTRODUCTION}

attern recognition is a field in computer studies that focuses on how machines interact with the environment, discover the different patterns according to a given rule and provide the desired output regarding the patters (Jain et al, 2000). A pattern in this case is a vaguely defined entity that bears a name such as a human face, or the image of a fingerprint. Pattern recognition may be either supervised or unsupervised. In supervised classification, the input pattern forms part of an already defined class while in unsupervised classification the pattern are assigned to an undefined category. Pattern recognition has a wide range of application including financial planning, data organization and recovery in multimedia databases and personal identification in biometrics (Murty \& Devi, 2011).

Three important aspects of pattern recognition that must be considered in designing pattern recognition systems include data input and preprocessing, representation of data and decisionmaking (Jain et al, 2000). The specific approaches and models for these aspects depend on the nature of the problem and how properly the problem is defined. When the problem is precisely defined, the recognition patter is likely to be more compact, resulting in a less sophisticated decision making technique. There are several methods applied in pattern recognition including neural networks, syntactic recognition, statistical recognition, and template matching (Jain et al, 2000, ). Apart from the neural network approach the other approaches are algorithmic, meaning they rely on programmed instructions to carry out their tasks (Liu et al, 2007, ). This paper will compare and contrast the algorithmic and neural network approaches to pattern recognition.

\section{ALGORITHMIC APPROACH VERSUS NEURAL NETWORK APPROACH}

To start with here is an Bruti Force Algorithm which is first Pattern matching algorithm , Here we compare a given pattern with the given substring

text $\mathrm{S}$ by trying each position one at a time.

$\mathrm{Wk}=\operatorname{Substring}(\mathrm{S}, \mathrm{k}$, Length $(\mathrm{P}))$

DOI : 10.5121/acij.2011.2617 
Here $\mathrm{S}$ is a string

$\mathrm{W}$ is a substring

$\mathrm{P}$ as length of substring

$\mathrm{K}$ is beginning with kth character.

Minimum value of $\mathrm{k}=1$.

Maximum value of $\mathrm{k}=$ Length(S)-Length(p)+1

First find $\mathrm{W}$ and compare $\mathrm{S}$ character by character. If all the characters are the same pattern matching is complete

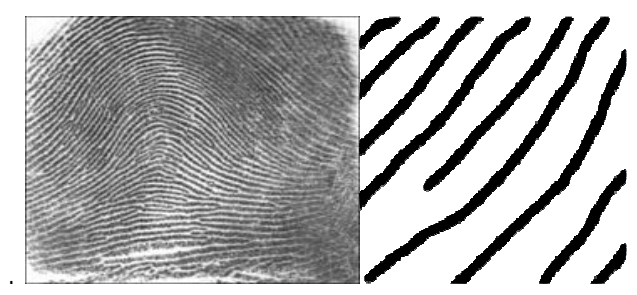

Fig.1

Similarly, in case of finger tips as given in fig. 1 one print is stored in the memory in the form of 0 and 1 . If the pixel is black then it is 1 else it is 0 . When the new finger tip comes across it is matched pixel by pixel on the basis of two dimensional matrix algorithms. If the pixel is black it gives 1 else 0 . Then both the 0's and 1's tables are matched if they are similar then finger tips are matched thus pattern recognition successful else unsuccessful.

Pattern recognition using neural networks via Boltzmann Machine(BM).The BM is a hetroassociative, pattern matcher that stores arbitrary spatial patterns $(\mathrm{Ak}, \mathrm{Ck}), \mathrm{k}=1,2, \ldots, \mathrm{m}$,

Here the kth pattern pair is represented by the vectors $A k=(a 1 k, \ldots$ ank $)$ and $c k=(c 1 k \ldots, c q k)$.

The BM is represented by the 3 layer topology.

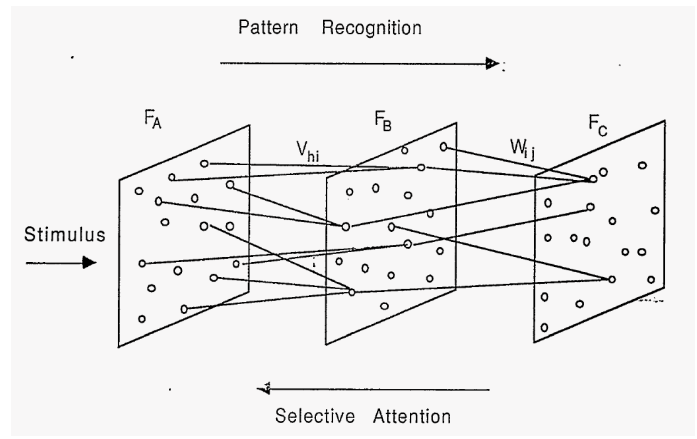

Fig.2

shown in fig 2. Where the Fa cells correspond to Ak's components and fc corresponds to ck components. The FA to FB interlayer connections are represented by Vhi, and all the FB to Fc inter-layer connections are indicated with wij. 
Let $\mathrm{F}$ be the set of all input patterns, $\mathrm{f}$, e.g. $\mathrm{f}=\mathrm{Ak}$. Let $\mathrm{T}$ be the parameter space of a family of $B M$, e.g. $t=T$, where $t=p(V h i, W i j)$. Therefore, given an input pattern $f=F$, one can find a $\mathrm{BM}$ such that the equilibrium distribution of this BM is the given pattern $\mathrm{f}$. Therefore, the input pattern, $\mathrm{f}$, is encoded into a specification of a BM, t. This mapping from $\mathrm{F}$ (pattern space) to $\mathrm{T}$ (parameter space of BM) defines the BM-transformation. BM-transformation encodes an input pattern into a relatively small vector which catches the characteristics of the input pattern. The internal space $\mathrm{T}$ is the parameter space of BM. The internal change rule of our system is defined by a formula.

$\Delta \mathrm{Ei}=\sum \mathrm{vhibi}+\sum \mathrm{wjibi}$

A stimulus pattern is presented to the first stage of the forward paths, the input layer FA, which consists of a two-dimensional array of receptor cells. The second stage of forward paths is called the hidden layer $\mathrm{Fb}$ The third stage of the forward paths is the recognition layer Fc. After the process of learning ends, the final result of the pattern recognition shows- in the response of the cells of FA. In other words, cells of the recognition layer Fc work as diagnostic cells; usually one cell is activated, corresponding to the category of the specific stimulus pattern. Cells in BM are featuring-extracting cells. Connections converging to these cells are variable and reinforced by learning (or training). After finishing the learning, those cells can extract features from the input patterns. In other words, a cell is activated only when a particular feature is presented at a certain position in the input layer. The features extracted by the cells are determined by the learning process. Generally speaking, local features, such as a line at a particular orientation, are extracted in the lower stages. More global features, such as part of a training pattern, are extracted in higher stages. Finally, each cell of the recognition layer at the highest stage integrates all the information of the input pattern; each cell responds to only one specific pattern. In other words, only one cell, corresponding to the category of the input pattern, is activated. Other cells respond to the patterns of other categories.

To better understand artificial neural computing it is important to know first how a conventional 'serial' computer and it's software process information. A serial computer has a central processor that can address an array of memory locations where data and instructions are stored. Computations are made by the processor reading an instruction as well as any data the instruction requires from memory addresses, the instruction is then executed and the results are saved in a specified memory location as required. In a serial system (and a standard parallel one as well) the computational steps are deterministic, sequential and logical, and the state of a given variable can be tracked from one operation to another. In comparison, ANNs are not sequential or necessarily deterministic. There are no complex central processors, rather there are many simple ones which generally do nothing more than take the weighted sum of their inputs from other processors. ANNs do not execute programmed instructions; they respond in parallel (either simulated or actual) to the pattern of inputs presented to it. There are also no separate memory addresses for storing data. Instead, information is contained in the overall activation 'state' of the network. 'Knowledge' is thus represented by the network itself, which is quite literally more than the sum of its individual components.

The main difference between the neural network and conventional approaches lies in the way they tackle the problem of pattern recognition (Minin, 2006,). The algorithmic approach used by conventional computers involves a host of instructions, translated into digital signals, which the computer follows to arrive at the solution. As such, the ability of the computer to solve a problem is limited to the programmer's understanding of the problem as well as their capability to solve it. This is one of the limitations of algorithmic approaches since they cannot solve problems that the programmer vaguely understands (Minin, 2006, ). 
Neural networks on the other hand, are capable of using analog signals and imitate the human brain in information processing (Ripley, 2007). The functional elements of a neural network are small processors called neurons that work hand in hand to solve a given problem (Ripley, 2007,). Neural networks are similar to the brain because they acquire knowledge to solve problems through learning as opposed to algorithmic approaches, which rely on programming. Another similarity of neural networks with the human brain is that information is stored in synaptic weights achieved through interconnection of neurons (Ripley, 2007,). The ability to learn from examples is one of the key features that make neural network approach unique. Hence, the examples chosen must be appropriate for the specific problem to be solved to minimize time wastage and network malfunction. This is particularly important since neural networks are not programmable. Since the network handles the problem independently, it is impossible to predict the outcome (Minin, 2006,).

On the contrary, the operation of conventional computers using algorithmic approaches is predictable. This is because algorithmic approach is based on a set of precise instructions, written in machine language that the computer can interpret and follow the instructions to arrive at the desired solution (Stergiou \& Siganos, 2011). Hence, the user can predict how the computer will deal with the problem. Neural networks are extremely useful in pattern recognition because of their ability to discover trends and patterns that algorithmic approaches cannot detect. When carefully trained, neuron networks can be regarded as experts in their specific areas of application hence can be relied on to predict future trends and answer complex logical questions (Minin, 2006).

Some of the strengths that neural networks possess include ability to retain the knowledge obtained through experience and adapt it to solve similar problems in future, excellent information organization skills, and real time operation achieved through parallel information processing. Additionally, the networks can still function even after partial destruction (Minin, 2006).

Despite these differences, both algorithmic and neural network approaches are complementary to each rather than being competitors. This is because some problems such as those involving calculations are best done using algorithmic approaches while others require neural networks (Stergiou \& Siganos, 2011 ). In addition, both approaches overlap especially in the underlying theory. Some of the procedures used in neural networks were derived from statistical pattern recognition technique. Largely both methods are branches of statistics (Petridis \& Kehagias, 1998).

Different categories of neural networks are available. The feed -forward and the Radial-Basis Function networks are the most common in pattern classification. The networks are arranged in layers with one-way connections between them (Jain et al, 200). Other networks in this category include the Kohonen-Network and the self -Organizing Map. Neural networks have gained popularity in pattern recognition because they do not depend much on the availability of specific knowledge for solving specific problems in pattern recognition as other methods do. Instead, the networks modify the knowledge learnt to suit the problem at hand and make decisions accordingly (Jain et al, 2000,).

The conventional approaches to pattern recognition are either parametric or non-parametric, in which the models applied are either fixed with few parameters or flexible respectively. Neural networks, on the other hand, insist on moderation of the two extremes, preferably models that are highly flexible but with limits and having a substantial number of parameter (Ripley, 2007).

There are many advantages and limitations to neural network analysis and to discuss this subject properly we would have to look at each individual type of network, which isn't necessary for this general discussion. In reference to backpropagational networks however, there are some specific issues potential users should be aware of.............??? Depending on the nature of the application and the strength of the internal data patterns you can generally expect a network to train quite well. This applies to problems where the relationships may be quite dynamic or non-linear. ANNs provide an analytical alternative to conventional techniques 
which are often limited by strict assumptions of normality, linearity, variable independence etc. Because an ANN can capture many kinds of relationships it allows the user to quickly and relatively easily model phenomena which otherwise may have been very difficult or impossible to explain otherwise.

\section{Conclusions}

Neural network approach to pattern recognition is the new area of research in computer science due to its potential to make the computer more useful in solving complex problems in pattern recognition. The main difference between this method and the conventional algorithmic approaches such as the syntactic and statistical methods is its ability to process information in a manner similar to the human brain. While algorithmic approaches require programming, neural networks operate without human intervention once the learning process is complete. The ability of neural networks to learn from examples and adapt the knowledge to solving numerous problems give this approach an edge over the algorithmic methods in discovering patterns that humans may not notice. Despite these differences, the two approaches complement each other. While algorithmic approaches are more useful in detecting arithmetic relationships, neural networks are more applicable in solving less analytical problems in pattern recognition.

\section{Acknowledgment}

The literary help and support rendered by Sh. Girish Aneja CA ...... and Dr R K Bhardwaj Associate Professor Dyal Singh College Karnal is fully acknowledged

\section{References}

Jain, K. A., Duin R. P. W. \& Mao J. (2000). Statistical pattern recognition: a review. IEEE Transactions on Pattern Analysis and Machine Intelligence, 22(1), 4-37.

Liu, D., Fei S., Hou Z., Zhang H. \& Sun C. (2007). Advances in neural networks-ISNN: $4^{\text {th }}$ international symposium on neural networks, ISNN 2007 Nanjing, China, June 3-7 proceedings, part 3. New York, NY: Springer

Minin, A. (2006). The neural network analysis. Retrieved July 13, 2011 http://www14.informatik.tumuenchen.de/konferenzen/Jass06/courses/2/presentations/minin_handout.pdf

Murty, N. \& Devi S. (2011). Pattern recognition: An algorithmic approach. New York: Springer.

Petridis, V., \& Kehagias, A. (1998). Predictive modular neural networks: Applications to time series. Boston: Kluwer Academic Publishers.

Ripley, B. D. (2007). Pattern recognition and neural networks. Cambridge: Cambridge University Press.

Stergiou, C. \& Signos D. (n.d). Neural networks. Retrieved July 13, 2011 http://www.doc.ic.ac.uk/ nd/surprise_96/journal/vol4/cs11/report.html\#Neural\%20networks\%2 0versus\%20conventional\%20computers 